\title{
Data Sharing Research of Intra-Day Security Check in Multi-Dispatching Center
}

\author{
LI Yan ${ }^{1,}$, , DING Qia ${ }^{1, b}$ and GE Rui ${ }^{2, c}$ \\ ${ }^{1}$ NARI Group Corporation (State Grid Electric Power Research Institute), NanJing 211106, China \\ ${ }^{2}$ National Electric Power Dispatching and Control Center, State Grid Corporation of China, BeiJing \\ 100031, China
}

aliyan2@sgepri.sgcc.com.cn, bdingqia@sgepri.sgcc.com.cn, coge-rui@sgcc.com.cn

\begin{abstract}
Keywords: Intra-day security check; Multi-Dispatching Center; Data sharing.
Abstract. Accurate power network data is the basis of grid security check. The plan data adjustment in different dispatching center influences each other. Regional balance was used in security check, based on fixed tie-line schedule, however, the influence of different dispatching center is ignored and power flow calculation result is affected. Instant and reliable data sharing in different dispatching center can provide more accurate plan data and improve power flow calculation result. This paper proposed a data sharing method in multi-dispatching center, increased immediacy and reliability of intra-day security check data sharing and support the coordinated operation in Multi-level dispatching center.
\end{abstract}

\section{Introduction}

With the approval of lots of UHV projects, the pace of construction of UHV power grid is accelerating. The scale of the power grid is developing rapidly and national interconnected power grid is basically formed. At present, the integration of power grid is greatly improved. From $500 \mathrm{kV}$ "hand in hand" interconnected power grid to the unified grid base on UHV, AC electrical contact closely, AC and DC switching capacity is huge. The impact between multi-level power grid is further enhanced. Grid coupling characteristics is highlighted and power grid characteristics is changing from the partition mode to the integration model. The rapid growth of power transmission capacity provides a solid and reliable hardware protection for resource optimization in a wide range and new energy consumption, but has also brought new challenges for safe operation of the grid.

The basis for the security check calculation of the grid is to obtain accurate grid operation data. For intra-day planning, the required planning data is similar to day ahead plan, mainly including load forecasting, power generation plan, tie-line plan, maintenance plan and section limit data ${ }^{[1]}$. Multi-dispatching center plan in the same grid and have mutual impact. It is used to adopt provincial balance model, the pre-determined tie-line plan was used as the provincial grid boundary conditions for the security check ${ }^{[2]}$, however, this method may produce some deviation. It is necessary to share data in multi-dispatching center and perform security check based on the whole network to ensure the calculation accuracy。

In the day ahead planning, provincial dispatching center transfer plan data to the higher-level dispatching center in files, step by step, the summary of all data is formed and then issued down to all dispatching center as the basis of security check for whole grid. However, Once the UHV AC/DC connection failure occurs, the transmission power of the transmitter is greatly reduced. If control strategies and power transmission plans is not timely adjusted, the failure may lead to chain reaction and risk of large-scale power loss during intra-day dispatching and operation will increase ${ }^{[3]}$. As a result, data sharing based on files is no longer adapt to data sharing performance requirements for multi-dispatching center intra-day planning adjustment. Literatures [4-6] put forward data sharing and exchange technology of their respective systems from different perspectives; Literature [4] put forward the idea of global sharing of power grid information, but did not give a concrete realization 
method; Literature [5-6] proposed a data sharing method between the horizontal systems in the regional power grid, but does not solve the large data sharing between the vertical multi-system.

\section{Fundamental}

To adapt the flexibility, reliability and immediacy requirements for wide-area sharing of data for intra-day security check, the paper presents a data source-exchange proxy service-client three-tier decoupling data sharing method. It can be used for intra-day joint dispatching and operation of multi-dispatching center to achieve efficient sharing of planned data and provide accurate data for security check to ensure safe and stable operation of power grid.

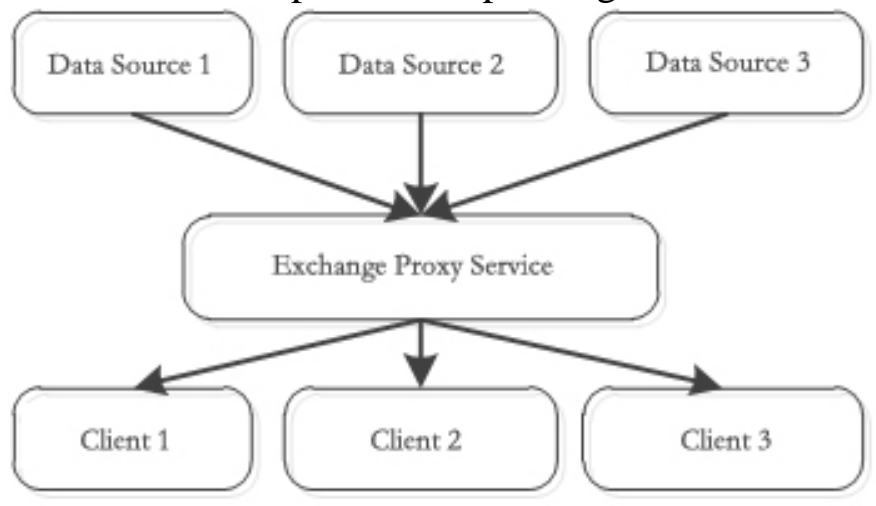

Figure 1 Three-tier topology of data sharing

In this method, data source and client are decoupled through the exchange proxy service. If only the exchange proxy service is deployed in client, the client can obtain data through exchange proxy service and no need to become the data source. The client can be extended independently of the data source, especially for many-to-many complex data sharing.

The exchange proxy service adopts the active/standby service mode, the active proxy service is responsible for all read and write operations, and the standby proxy service only synchronizes the data with the active proxy service. The active proxy service makes multiple backups of the data for itself and the standby proxy service; If the standby proxy service fails, the historical data of the active proxy service is synchronized from another standby proxy service; If the active proxy service fails, other standby proxy service becomes the new active proxy service to continue the service, improves the reliability of data sharing.

After the proxy service receives the data, the received data is indexed and stored in the cache entity file. The active proxy service indexes the received data and then stores it in the cached entity file and improves the data access efficiency through parallel access. Compared to the general data exchange using memory or random file cache to retain, append mode is used here to reduce the disk retrieval costs, improve the data access efficiency and ensure data reliability.

\section{Example}

In this paper, the application of the data sharing method is described in detail with the application scenario of the security check data sharing in the national, branch and provincial dispatching centers. 


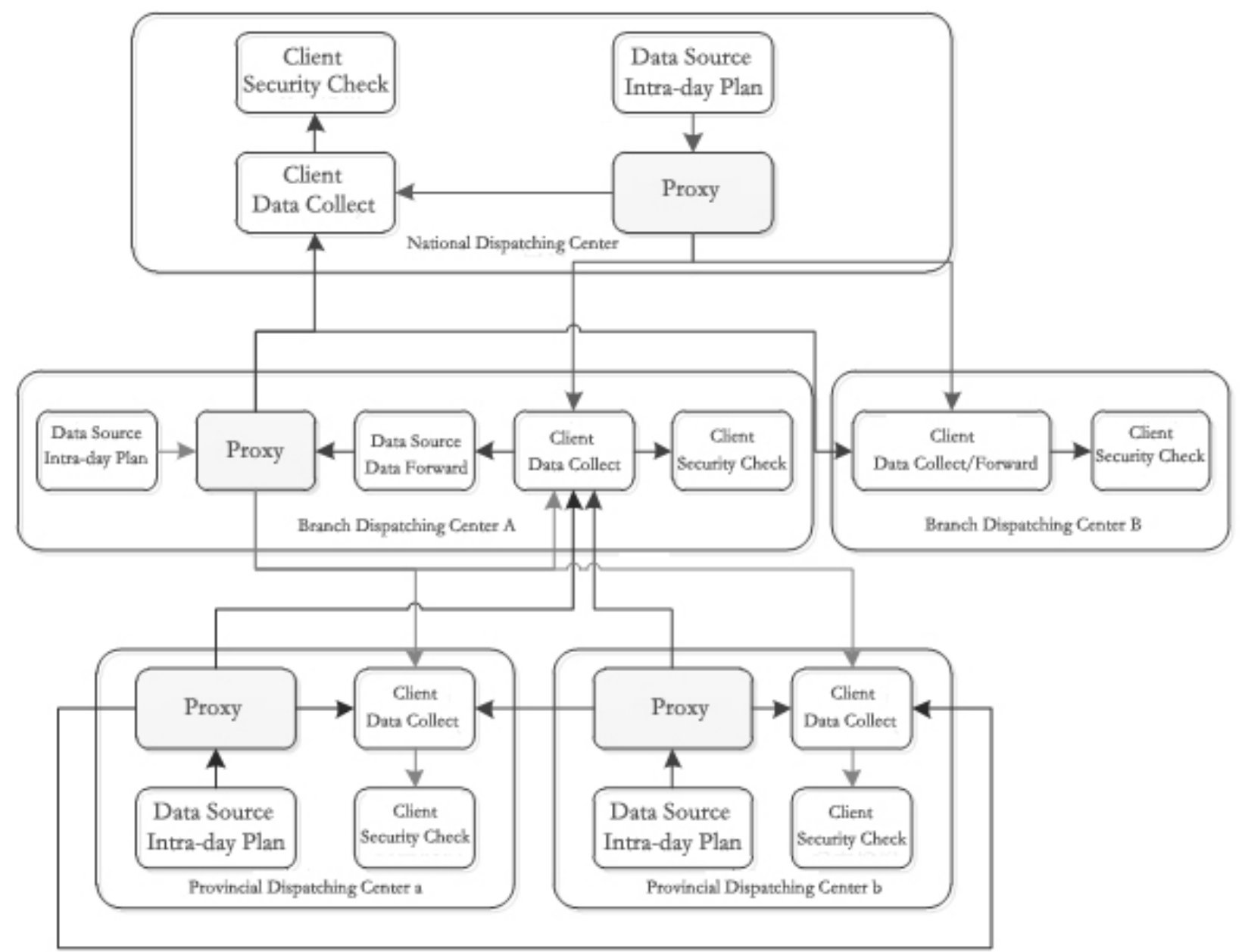

Figure 2 Multi-Dispatching Center Security Check Data Sharing Process Based on Proxy Service

Multi-dispatching center data sharing used hierarchical exchange method. Branch dispatching center plays a connecting role. The process is described as follows.

1) Create page cache entity files according to maximum parallel access and index one as the active proxy service, others as standby proxy service. All exchange proxy services exist in permanent processes and the page cache entity file is the carrier of the exchange proxy service.

2) National, branch and provincial dispatching centers separately perform data preparation, gathering local plan data, including load forecasting, power generation plan, tie-line plan, maintenance plan and section limit data, communicate with local exchange proxy service and push the data.

3 ) After the proxy service in national, branch and provincial dispatching centers receives the data, the received data is indexed and stored in the cache entity file。

The active proxy service caches the received data in append mode. The active proxy service makes multiple backups of the data for itself and the standby proxy service; If the standby proxy service fails, the historical data of the active proxy service is synchronized from another standby proxy service; If the active proxy service fails, other standby proxy service becomes the new active proxy service to continue the service。

In order to ensure the reliability of data, the active proxy service is responsible for all read and write operations, and the standby proxy service only synchronizes the data with the active proxy service. Compared to the general data exchange using memory or random file cache, append mode is used here to reduce the disk retrieval costs, improve the data access efficiency and ensure data reliability. The active proxy service and standby proxy service return finish flag to data source after data is cached.

4) Data summary in branch and provincial dispatching center is defined as data source. Active proxy service and standby proxy service can be located in wide area network by IP address. The client sends 
data request to local proxy service using data index, retrieve data, record the retrieved data index, and then enter the next cycle.

5 ) After client dispatching center get necessary intra-day plan data, security check is performed according to calculation cycle

\section{Conclusions}

1) This paper proposed a data source-exchange proxy service-client three-tier decoupling data sharing method, clients can be easily extended by configuration, without modifying the method to realize data sharing in multi-dispatching centers.

2) The exchange proxy service adopts the active/standby service mode and do persistent processing to data content to ensure the reliability when network or part of the component fail.

3 ) Improve the data access efficiency through parallel access. Compared with the file-based sharing mode, sharing efficiency is greatly improved.

4) Efficient data sharing of intra-day security check in multi-dispatching center save more time for security analysis and schedule adjustment and provide technical support for safe operation of the grid.

\section{Acknowledgements}

This work was financially supported by Science and Technology Project of State Grid Corporation "Research on Optimization and Adjustment Technology for Intraday Generation Scheduling of Mutual Support between Regional Power Grid Considering Large-scale Power Loss"

\section{References}

[1] XingJing, LuFeng, ZhangJuan. Design and Implementation of Quantization Safety Verification System for Jibei Grid[J].North China Electric Power,No.6 2016.

[2] XuDan, CaiZhi, LiXiaolei. Multi-level Coordination Plan Model Based on Constrained Adj ustmen[J].Automation of electric power systems, Vol.39 No.1 Jan.10,2015.

[3] XuDan, GeRui, CaiZhi. Optimization and Adjustment Method for Intraday Generation Scheduling of Regional Power Grid Considering Large-scale Power Loss[J].Automation of electric power systems, Vol.41 No.7 Apr.10,2017.

[4] ChengShijie, LiXingyuan, ZhangZhizhe. Entire-grid-area Information-sharing and Integrated Applications in United Information System for Smart Grid[J].Proceedings of CSEE,2011,31(1):8-14. [5] WangWeiguo, DaiWei, WanLei. Discussion on Data Sharing Mode in Electric Power Network Dispatching Automation System[J].Automation of electric power systems, Vol.29 No.4 Feb.25,2005 [6] FanWeiqin. Practice of Building Regional Power Dispatching Integrated Data Platform[J].Automation of electric power systems,2010,34(2):113-115. 